\title{
Personal Recognition Using Single-Sensor Multimodal Hand Biometrics
}

\author{
Andreas Uhl and Peter Wild \\ University of Salzburg, Department of Computer Sciences, A-5020 Salzburg, Austria \\ $\{$ uhl,pwild $\} @$ cosy.sbg.ac . at
}

\begin{abstract}
Single-sensor approaches to multimodal biometric authentication targeting the human hand in multiple-matcher scenarios provide higher security in terms of accuracy and resistance to biometric system attacks than unimodal systems. This paper introduces a novel multimodal hand biometric system using palmar images acquired by a commercially available flatbed scanner. Hence, the presented approach to personal recognition is independent of specific biometric sensors, such as fingerprint readers or palmprint scanners. Experimental results with a minimum half total error rate of $0.003 \%$ using a database of 443 hand images will illustrate the performance improvement when hand-geometry, fingerprint and palmprint-based features are combined.
\end{abstract}

\section{Introduction}

Biometrics facilitates authentication tasks by means of independence of knowledge and tokens (which may be passed on fraudulently), permanent and singular features (which may hardly be lost without intention nor forgotten like passwords), and low mean transaction times for access control. Biometric authentication systems in verification mode (comparison with a claimed identity) should not exceed $0.1 \%$ False Match Rate (FMR) at $0.1 \%$ False Non-Match Rate (FNMR) according to [1. In order to bridge the gap between required rates and performance of current unimodal matchers, multimodal systems combine different modalities. The contribution of this paper is a novel single-sensor multibiometric system integrating common modalities related to the human hand:

- Hand geometry using a feature targeting widths of single fingers;

- Fingerprint extracting local-level textural features at fingertips;

- Palmprint focusing on textural properties of the human palm tracking principal lines and wrinkles;

The novelty of the presented approach lies in the extraction of all features out of a simple palmar scan using a common flatbed scanner, which will be shown to produce very accurate results. Originally different sensing devices have been developed for each of the integrated modalities. The presented approach is motivated by Kumar et al. 2. However, their results only refer to randomly paired samples from multiple databases indicating an improvement of the best single 
biometric trait by means of Equal Error Rate (EER) performance of roughly $40 \%$ (yielding a total EER of $3.53 \%$ ). Recently, an implemented solution extracting samples out of a single acquired input signal based on multispectral image processing has been presented by Rowe et al. 3. They report no errors on their dataset of 50 volunteers for fingerprint score fusion using the Sum Rule [4] and a significant performance improvement if ring finger and palmprint scores are combined. A significant drawback of their presented solution is the requirement of special image sensors based on multispectral technology. Using document scanners designed for large markets instead has several advantages:

- Availability: according to a study in [5], every third US household held a document scanner by 2001 expecting a market saturation by 2006 .

- Reproducibility: sensor requirements of 500-dpi 8-bit grayscale at reasonable scanning speed are largely covered by existing scanners.

- Sensor independence: results of the employed HP Scanjet 3500c model, which is a Charge Coupled Device (CCD) scanner placed 2002 in the low-cost workgroup market segment [5], are most likely to remain stable or even improve when faster and more accurate hardware is employed.

- Cost: when hardware for large markets is used, acquisition and upkeep costs are minimized.

The structure of this paper follows the information flow in common biometric systems. After a description of sensor and preprocessing steps in Sect. 2, feature extraction and matching techniques are introduced in Sect. 3. System performance is assessed by experimental evaluation in Sect. 4. Results are discussed comparing single and combined modalities. Section 5 forms the conclusion.

\section{Sensing and Preprocessing}

For experiments we used a HP Scanjet 3500c scanner in 500-dpi and 8-bit grayscale mode. Users were free to choose an arbitrary placement when inserting their hand through a hole in a box containing the sensor (to minimize environmental light, see Fig. 10 as long as their fingers did not touch each other, i.e. the system is peg-free. Thus, preprocessing is essential to provide each extractor with normalized data and to achieve rotational and translational invariance.

After input acquisition, the hand object is localized within the $4250 \times 5850$ image with respect to the smallest circumscribed axis-aligned rectangle. The $a \times b$ hand image $H(x, y)$ with $1 \leq x \leq a, 1 \leq y \leq b$ is segmented using a fast version of Otsu's thresholding [6]. Arm parts in the input image are removed iteratively. Within every step of the iteration, the hand image is aligned using statistical moments estimating the inclination of the best-fitting ellipse centered in the center of mass, a technique from face-detection [7. Then, the horizontal wrist line separating arm and palm is estimated by a top-down scan and the corresponding arm part is masked in the input image. This procedure is iterated 3 times to obtain a stable segmentation.

Despite its application in many hand biometric systems, such as in 89, ellipse-fitting is found to produce imperfect alignment for different spreadings of 


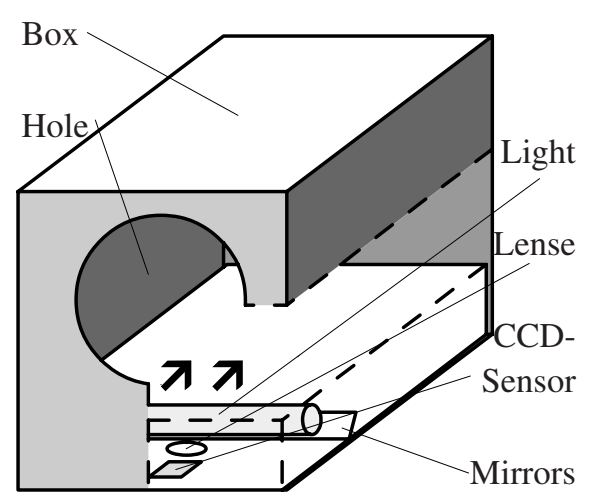

Fig. 1. Employed flatbed sensor

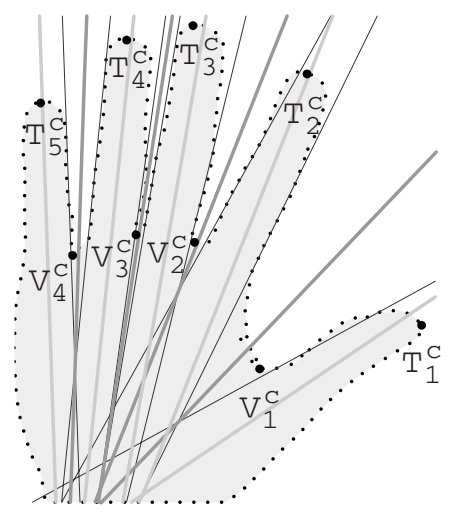

Fig. 2. Salient point refinement for hands

fingers. Instead, we employ a predefined hand coordinate system, an alignment procedure frequently used in palmprint recognition [10. Let $\boldsymbol{V}_{i}$ with $1 \leq i \leq 4$ denote the finger valleys of a right hand in counter-clockwise order and let $\boldsymbol{T}_{j}$ with $1 \leq j \leq 5$ track positions of finger tips of thumb, index, middle, ring and small finger. Then the origin of the new coordinate system is defined as the position of the inter-finger valley $\boldsymbol{V}_{3}$ and its y-axis is the line parallel to the least-squares approximation of the outer palm boundary through $\boldsymbol{V}_{3}$. The palm boundary is defined as the subsequence $\left\langle\boldsymbol{C}_{s}, \ldots, \boldsymbol{C}_{e}\right\rangle$ of the counter-clockwise contour sequence $\left\langle\boldsymbol{C}_{0}, \ldots, \boldsymbol{C}_{z}\right\rangle$ within a range defined by the average length $l$ of fingers for the given hand:

$$
\begin{aligned}
& s=\max \left\{k \in\{0, \ldots, z\}:\left\|\boldsymbol{C}_{k}-\boldsymbol{T}_{5}\right\| \leq\left\|\boldsymbol{V}_{4}-\boldsymbol{T}_{5}\right\|\right\} ; \\
& e=\max \left\{k \in\{0, \ldots, z\}:\left\|\boldsymbol{C}_{k}-\boldsymbol{C}_{s}\right\| \leq l\right\} .
\end{aligned}
$$

Candidates for salient points (i.e. finger peaks and valleys) are typically extracted from the silhouette contour by finding local extrema of the radial distance function 11] with respect to a reference point at the wrist. In our approach, peak candidates are refined by intersecting the major axis of the best-fitting ellipse for each individual finger (using candidate valleys as separators between fingers) with the contour. Finger valley refinement comprises an approximation of the proximal finger boundary by straight lines on each side and an intersection of bisecting lines between fingers with the contour, see Fig. 2 .

\section{$3 \quad$ Feature Extraction and Matching}

We employ four different geometric and texture-based algorithms:

- Shape: divides each finger into slices of equal height and keeps track of the local finger width for each slice (see [12] for an application on footprints). 
- Minutiae: identifies minutiae points within a finger-axis-aligned rectangle at finger tips using NIST's minutiae extraction software 13 .

- Palmprint: extracts variances of 144 overlapping blocks in the palmprint image after edge detection (method introduced by Kumar et al. [9]).

- Eigenpalms and Eigenfingers: projects each finger and palm onto the eigenspace spanned by the most significant principal components (see [14]).

The employed scenario represents a score level fusion approach (i.e. matching scores rather than feature vectors or decision vectors of independent algorithms are combined) operating in multiple-matcher configuration. Whereas more independent levels of integration exist (such as multiple biometrics or multiple sensors for example), information can be expected to be largely independent, since employed algorithms operate at:

- different resolutions: Shape, Minutiae and Palmprint require the full 500-dpi input signal, Eigenpalms and Eigenfingers are extracted at $100 \mathrm{dpi}$.

- different parts of the hand: Minutiae operates on each of the five finger tips, Eigenpalms and Palmprint extract information from the textural palm area while Eigenfingers and Shape concentrate on fingers.

Each individual feature extractor contributes its extracted representation to a common augmented feature vector. This template is stored together with an identifying key in a preceding enrollment mode and compared with the stored reference in authentication mode 4]. For matching, augmented feature vectors are decomposed into feature-dependent parts and matching scores are generated for each of the employed features.

\subsection{Shape Feature}

The Shape feature depicted in Fig. 3 targets hand geometry measurements extracting local finger widths from the hand contour. Each finger is rotated upright
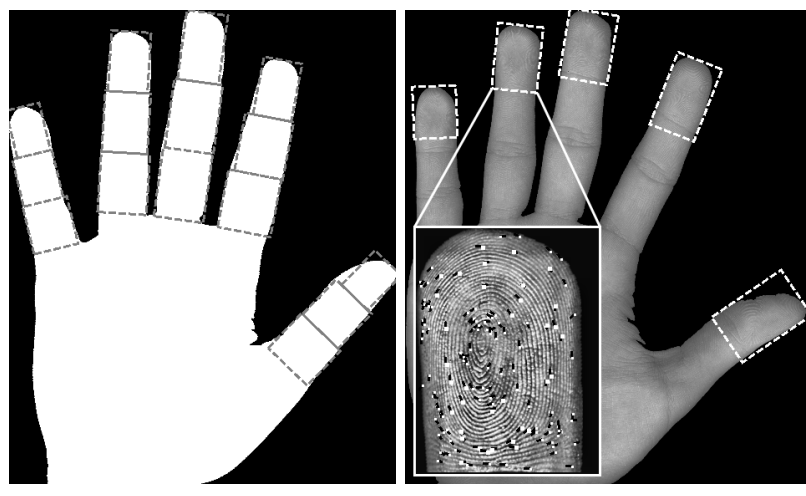

Fig. 3. Shape feature
Fig. 4. Minutiae feature

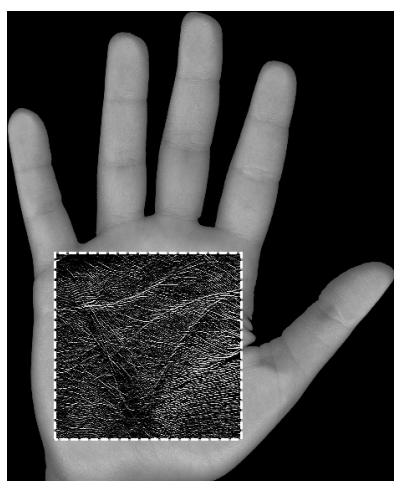

Fig. 5. Palmprint feature 
using moments [7] and cropped at the adjacent finger valley closer to the tip. For each of $c=3$ slices $S_{1}, \ldots S_{c}$ of equal height covering the finger we determine the object's average width $w\left(S_{n}\right), 1 \leq n \leq c$, with respect to the y-monotone contour extracted using a left-right scan. Matching is based on Manhattan metric.

\subsection{Minutiae}

We extract position, orientation and quality of termination and bifurcation points in the outermost structural part of the epidermis employing the NFIS2 [13] extraction software mindtct on the enhanced fingerprint image. While sensing regions may also be defined for other parts of the human hand (see [3] for results on metacarpal skin), we extract each fingerprint image as a $w \times \frac{h}{3}$ (and $w \times \frac{h}{2}$ for the thumb, respectively) rectangular area aligned with respect to the major axis of the finger circumscribed by its $w \times h$ sized bounding box. Since flatbed optical images exhibit low contrast between ridges and valleys, Contrast-limited adaptive histogram equalization described in [15] has been employed. Minutiae pairing and matching is executed using the NFIS2 bozorth3 matcher. Matching results of individual fingerprints are combined using the Max Rule (returning the highest score of all fingers) [4].

\subsection{Palmprint Feature}

After localization of the hand-axis aligned square palmprint region of size $l$ (being the average finger length) centered in $\boldsymbol{P}=\left(0,-\frac{7}{10} l\right)$ with respect to the introduced hand coordinate system, edges are detected using a $7 \times 7$-Prewitt filter, see Fig. 5. Features are extracted as variances within blocks of size $24 \times 24$ pixels within a downscaled and normalized $300 \times 300$ version of the palm, as proposed in 9]. The Palmprint matcher computes a normalized squared Euclidian distance between feature vectors.

\subsection{Eigenpalms and Eigenfingers}

Eigenpalms and Eigenfingers (Figs. 677) are derived from Eigenfaces introduced by Turk and Pentland [16] and are based on the Karhunen-Loeve transform converting image vectors of single fingers or palms into a space spanned by the most significant components calculated from a set of training images. After subtracting the average image vector $\boldsymbol{A}$ for each type of training image $(256 \times 256$ palm images, $128 \times 384$ index, middle, ring fingers and $128 \times 256$ thumb and little finger images), the $d=25$ eigenvectors $\boldsymbol{E}_{m}, 1 \leq m \leq d$ with largest eigenvalues are computed from the covariance matrix of normalized samples. For each image type feature extraction corresponds to (a) extraction of finger or palm image $\boldsymbol{I}$; (b) normalization with respect to the average image vector $\boldsymbol{A}$ and; (c) projection onto the corresponding space spanned by $\boldsymbol{E}_{m}$ and storage of projection coefficients as feature components. The corresponding matcher is based on Manhattan metric, matching scores of individual eigenspaces are combined using the Product Rule. 


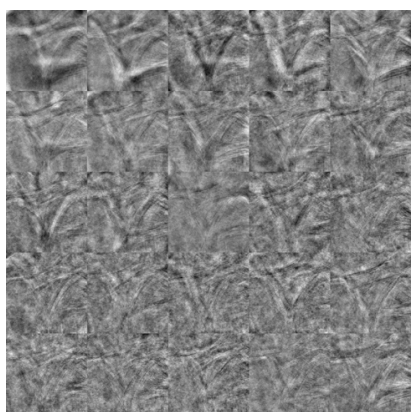

Fig. 6. Eigenpalms

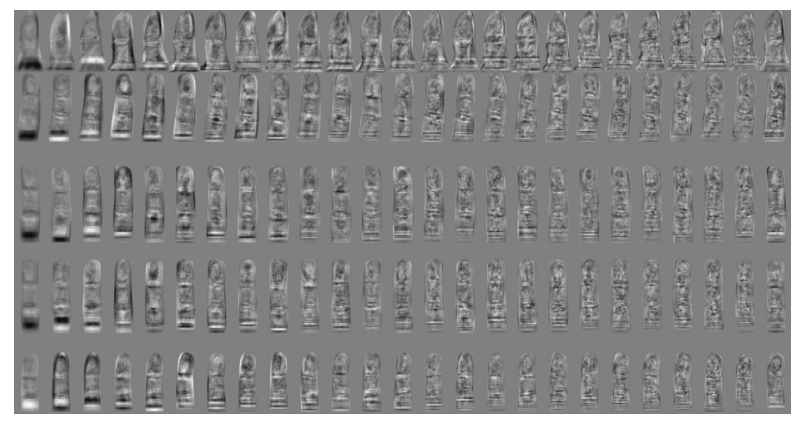

Fig. 7. Eigenfingers

\section{Performance Evaluation}

We evaluate verification performance of single and combined features in the introduced hand biometric system by means of Receiver operating characteristics depicted in Fig. 8 and indicators given in Table 1 including MinHTER performance, i.e. the global minimum of the Half total error rate function [17]:

$$
\operatorname{HTER}(t):=\frac{\operatorname{FMR}(t)+\operatorname{FNMR}(t)}{2} .
$$

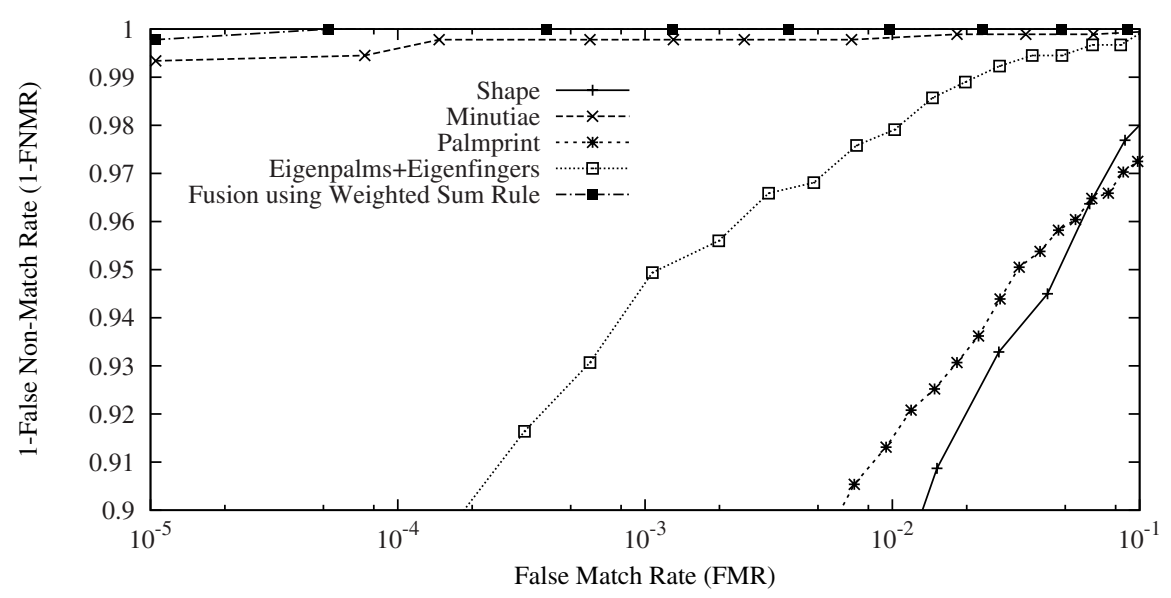

Fig. 8. Receiver operating characteristics

\subsection{Experimental Database}

For testing purposes a database of 443 right-hand samples of 71 males and 15 females recorded at the Department of Computer Sciences, University of 
Table 1. Verification results for the employed algorithms

\begin{tabular}{llll}
\hline Algorithm & MinHTER & ZeroFMR & ZeroFNMR \\
\hline Shape & $4.7 \%$ & $70.74 \%$ & $25.53 \%$ \\
Minutiae & $0.12 \%$ & $1.1 \%$ & $16.44 \%$ \\
Palmprint & $4.1 \%$ & $36.19 \%$ & $100 \%$ \\
Eigenpalms + Eigenfingers & $1.44 \%$ & $15.29 \%$ & $10.72 \%$ \\
Fusion using Weighted Sum Rule & $0.003 \%$ & $0.33 \%$ & $0.005 \%$ \\
\hline
\end{tabular}

Salzburg, Austria has been employed. Samples were acquired within a timespan of 15 minutes per person. All users were allowed to wear rings or watches. Failure to Acquire (FTA) exceptions occurred in $0.9 \%$ of the cases.

\subsection{Verification Performance}

When executing a cross-comparison of available templates resulting in 909 genuine and 95232 imposter comparisons, the best matching results with respect to MinHTER performance are returned by the Minutiae feature with $0.12 \%$. Especially for high-security environments, where low FNMR at almost zero FMR is demanded, this feature outperforms all other tested algorithms. Rates for fusionbased Eigenpalms + Eigenfingers-based recognition are an order of magnitude higher with a MinHTER value of $1.44 \%$. Low resolution requirements of $100 \mathrm{dpi}$, fast matching speed and reasonable matching performance especially for applications demanding high convenience are special characteristics of this feature. However, recently published results by Cheung et al. [18] indicate that recognition rates for Eigenpalms tend to degrade significantly in case of larger time lapses between recordings and twins. The Palmprint feature provides less accurate results at $4.1 \%$ MinHTER, which lies in the order of reported error rates in [9]. Geometry-based Shape could not outperform texture-based features with 4.7\% MinHTER. If results from all modalities are combined using Weighted Sum Rule fusion, a significantly higher performance of $0.003 \%$ MinHTER can be achieved. An optimum configuration of weights (with respect to a step size of 0.01 ) was found at 0.10 for Shape, 0.17 for Palmprint, 0.06 for Eigenpalms + Eigenfingers, and 0.67 for Minutiae. Although the eigenspace-based features exhibit the second best MinHTERperformance, their weight within the best common feature is rather small. This behavior might possibly be caused by high correlation between Eigenfingers and Shape, since Eigenfingers are sensitive to both shape and textural information.

\section{Summary}

We have proposed a new multimodal flatbed-scanner-based approach to personal recognition with hands integrating hand geometry, fingerprint and palmprintbased algorithms in a multiple-matcher scenario. The performance of the introduced system has been assessed using a custom medium-sized database. 
Considering the overall performance in verification mode a MinHTER of $0.003 \%$ could be achieved with fusion at matching score level, outperforming each of the individual features. Generally, texture-based features produced better results (Minutiae with $0.12 \%$ MinHTER, followed by Eigenpalms + Eigenfingers with $1.44 \%$ and Palmprint with $4.1 \%$ ) than geometry-based Shape with $4.7 \%$ MinHTER. Future topics of interest comprise larger time-lapses between recordings, studies on twins and an evaluation based on more different flatbed sensors.

\section{References}

1. Jain, A.K., Pankanti, S., Prabhakar, S., Hong, L., Ross, A.: Biometrics: A grand challenge. In: Proceedings of the 17th International Conference on Pattern Recognition, pp. 935-942 (2004)

2. Kumar, A., Zhang, D.: Combining fingerprint, palmprint and hand-shape for user authentication. In: Proceedings of the 18th International Conference on Pattern Recognition, pp. 549-552 (2006)

3. Rowe, R.K., Uludag, U., Demirkus, M., Parthasaradhi, S., Jain, A.K.: A multispectral whole-hand biometric authentication system. In: Proceedings of Biometrics Symposium, pp. 1-6 (2007)

4. Ross, A.A., Jain, A.K.: Information fusion in biometrics. Pattern Recognition Letters 24, 2115-2125 (2003)

5. InfoTrends, Inc.: Scanner market reaches maturity - penetration nearing one third of U.S. PC households (2001), http://www.infotrends.com/ public/Content/Press/2001/06.19.2001.html

6. Liao, P.S., Chen, T.S., Chung, P.C.: A fast algorithm for multilevel thresholding. Journal of Information Science and Engineering 17(5), 713-727 (2001)

7. Sobottka, K., Pitas, I.: Extraction of facial regions and features using color and shape information. In: Proceedings of the 13th International Conference on Pattern Recognition, pp. 421-425 (1996)

8. Yörük, E., Dutagaci, H., Sankur, B.: Hand biometrics. Image and Vision Computing 24(5), 483-497 (2006)

9. Kumar, A., Wong, D.C.M., Shen, H.C., Jain, A.K.: Personal verification using palmprint and hand geometry biometric. In: Proceedings of the 4th International Conference on Audio- and Video-Based Biometric Person Authentication, pp. 668678 (2003)

10. Zhang, D.: Palmprint authentication. Kluwer Academic Publishers, Dordrecht (2004)

11. Yörük, E., Konukoglu, E., Sankur, B., Darbon, J.: Shape-based hand recognition. IEEE Transactions on Image Processing 15, 1803-1815 (2006)

12. Uhl, A., Wild, P.: Personal identification using eigenfeet, ballprint and foot geometry biometrics. In: Proceedings of the IEEE First International Conference on Biometrics: Theory, Applications, and Systems, pp. 1-6 (2007)

13. NIST: Fingerprint Image Software 2 (2004), http://fingerprint.nist.gov/NFIS

14. Ribaric, S., Fratric, I.: A biometric identification system based on eigenpalm and eigenfinger features. IEEE Transactions on Pattern Analysis and Machine Intelligence 27(11), 1698-1709 (2005)

15. Zuiderveld, K.: Contrast limited adaptive histogram equalization. In: Graphics gems IV, San Diego, CA, USA, pp. 474-485. Academic Press Professional, Inc, London (1994) 
16. Turk, M., Pentland, A.: Eigenfaces for Recognition. Journal of Cognitive Neuroscience 3(1), 71-86 (1991)

17. Pierrot, J., Lindberg, J., Koolwaaij, J., Hutter, H.P., Genoud, D., Blomberg, M., Bimbot, F.: A comparison of a priori threshold setting procedures for speaker verification in the CAVE project. In: Proceedings of the International Conference on Acoustics, Speech, and Signal Processing, pp. 125-128 (1998)

18. Cheung, K.H., Kong, A., Zhang, D., Kamel, M., You, J.: Does eigenpalm work? a system and evaluation perspective. In: Proceedings of the 18th International Conference on Pattern Recognition, pp. 445-448 (2006) 